\title{
DILATIONS ASSOCIATED TO FLAT CURVES
}

\author{
STEPIIEN WAINGER
}

I would like to give an exposition of recent work of Tony Carbery, Mike Christ, Jim Vance, David Watson, and mysclf concerning Hilbert transforms and Maximal functions along curves in $R^{2}$ [CCVWW]. Thus we let $\Gamma(t)=$ $(t, \gamma(t))$ be a curve in $R^{2}$ with $\gamma(0)=\gamma^{\prime}(0)=0$.

For a function $f$ in $C_{0}^{\infty}\left(R^{2}\right)$, we set

$$
H_{\Gamma} f(x)=\int_{-1}^{1} f(x-\Gamma(t)) \frac{d t}{t},
$$

and

$$
M_{1} f(x)=\sup _{0<k \leq 1} \frac{1}{h} \int_{0}^{h}|f(x-\Gamma(t))| d t .
$$

We are interested in the problem of obtaining estimates of the form

$$
\left\|H_{\Gamma} f\right\|_{L^{r}} \leq A(p, \Gamma)\|f\|_{L^{p}}
$$

and

2)

$$
\left\|\mathcal{M}_{\Gamma} f\right\|_{L_{P}} \leq A(p, \Gamma)\|f\|_{L^{2}} .
$$

Positive results have been known for a long time for 1) and 2) under an appropriate curvature hypothesis. The curvature hypothesis is that $\gamma^{(k)}(0) \neq 0$ for some $k \geq 2$. Thus if

$$
\Gamma(t)=\left(t, t^{k}\right) \quad k \geq 2, k \text { a positive integer, }
$$

or

4)

$$
\Gamma(t)=\left(t, t^{k}-t^{k+1}\right) \quad k \geq 2, k \text { a positive integer, }
$$

the curvature condition is satisfed. If

$$
\Gamma(t)=\left(t, e^{-1 / t^{2}}\right)
$$

the curvature condition is not satisfied. 
An important property of curves for which the curvature hypothesis holds is that it is almost homogeneous. We say $\Gamma(t)$ is homogeneous if there exists a group of linear transformations $A(\lambda)$, defined for $\lambda>0$, such that,

$$
\Gamma(\lambda t)=A(\lambda) \Gamma(t)
$$

7)

$$
A(\lambda \mu)=A(\lambda) A(\mu)
$$

and

8)

$$
A(\lambda) x \longrightarrow 0 \text { as } \lambda \longrightarrow 0 \text { for every } x
$$

Thus $\Gamma(t)=\left(t, t^{k}\right)$ is homogeneous with

$$
A(\lambda)=\left(\begin{array}{cc}
\lambda & 0 \\
0 & \lambda^{k}
\end{array}\right)
$$

and $\Gamma(t)=\left(t, t^{k}-t^{k+1}\right)$ is almost homogeneous in that

$$
\Gamma(\lambda t)=A(\lambda) \Gamma(t)+\text { small error. }
$$

In 3) and 4) above the dilations $A(\lambda)$ are staring one in the face. The question we are interested in is whether or not there could be a useful farnily of dilations for curves in which the curvature condition fails, a curve like

$$
\Gamma(t)=\left(t, e^{-1 / t^{2}}\right) .
$$

In discussing this question, it is well to keep in mind another exarnple of a homogeneous curve, namely

9)

$$
\Gamma(t)=(t, t \log |t|)
$$

Here

$$
A(\lambda)=\left(\begin{array}{cc}
\lambda & 0 \\
\lambda \log \lambda & \lambda
\end{array}\right) .
$$

Thus if wc wish to have a theory that includes the example 9), we would not want to take

$$
A(\lambda)=\left(\begin{array}{cc}
\lambda & 0 \\
0 & \gamma(\lambda)
\end{array}\right)
$$

in general.

Before describing the family of dilations that we found, let us try to decide what we might hope to prove using them.

Let us assume that $\gamma(t)$ is odd and convex for $t>0$. Then most known results are expressible in terms of a functional $h(t)$,

$$
h(t)=t \gamma^{\prime}(t)-\gamma(t)
$$

Geometrically $h(t)$ represents the distance from the origin to the $y$-intercept of the line tangent to $\Gamma$ at $\Gamma(t)$.

We then have the following Theorem: 
Theorem A. Assume $\gamma(t)$ is odd and convex for $t>0$, then

$$
\left\|H_{\Gamma} f\right\|_{L^{2}} \leq A\|f\|_{L^{2}}
$$

if and only if

$$
h(c t) \geq 2 h(t) \text { for all } t>0
$$

for some $c>0$. Also if

$$
h(c t) \geq 2 h(t)
$$

for some $c>0$ and all $t>0$,

$$
\left\|\mathcal{M}_{\Gamma} f\right\|_{L^{2}} \leq c\|f\|_{L^{2}} .
$$

See [NVWW1] and [NVWW2].

Further it is known that 10) does not suffice for 1) or 2) for all $p, 1<p<\infty$. See $[C C N W W]$ and $[C 1]$. Using the family of dilations we construct we are able to prove the following:

Theorem B. Assume $\gamma(t)$ is add and convex for $t>0$. Then if for some $\varepsilon>0$,

$$
\begin{aligned}
& h^{\prime}(t) \geq \varepsilon \frac{h(t)}{t}, \\
& \left\|H_{\Upsilon} f\right\|_{L^{p}} \leq A(p, \Gamma)\|f\|_{L^{p}}, 1<p<\infty,
\end{aligned}
$$

and

$$
\left\|\mathcal{M}_{\Gamma} f\right\|_{L^{D}} \leq A(p, \Gamma)\|f\|_{L^{p}, 1}<p<\infty,
$$

(Other sufficient conditions are given in [CCCDRVWW] and [CW].)

We may view 11) as an infinitesinal version of 10). In particular 11) implies $10)$.

It turns out that the family of dilations we use are

$$
B(\lambda)=\left(\begin{array}{cc}
\lambda & 0 \\
\gamma(\lambda) & h(\lambda)
\end{array}\right)
$$

Note that in the case of the curve

$$
\Gamma(t)=(t, t \log |t|)
$$

$B(\lambda)$ and $A(\lambda)$ are the same. In the case that

$$
\Gamma(t)=\left(t, t^{k}\right)
$$


$A(\lambda)$ and $B(\lambda)$ are not the same, but they are equivalent in the sense that if $K$ is an open convex set containing the origin in its interior, there is another such set $K_{1}$ such that

$$
A(\lambda) K_{1} \subset B(\lambda) K
$$

and visa versa.

The dilations $B(\lambda)$ do not form a group, that is condition 7) is violated. However, one can dispense with 7) if one proves

$$
\left\|B(s)^{-1} B(t)\right\| \leq C\left(\frac{t}{s}\right)^{\epsilon}
$$

for some positive $\varepsilon$. It turns out that 10) implies 12).

The dilations are used in the proof of Theorem $B$ in 2 ways. The first application is to obtain uniformy decay estimates for measures supported on $\Gamma(t)$, and the second is to be able to develop a Catderon-Zygmund theory. The Calderon-Zygmund theory gives rise to a Littlewood Paley Theory from which Theorem $\mathrm{B}$ can be derived using ideas developed in [DR] and [NSW]. The Calderon Zygmund theory is developed by using a variant of the argument of Coifman and Weiss concerning spaces of homogeneous type [CW]. Here we shall concetrate on the decay of the Fourier trasnform of measures supported on $\Gamma$. We refer the reader to [CCVWW] for the Calderon Zygmund theory, Littlewood Paley arguments and the rest of the proof of Theorem B.

We define measures $d \mu_{n}$ supported on $\Gamma$ as follows: For a test function $\phi$

$$
d \mu_{n}(\phi)=2^{n} \int_{2^{-n}}^{2 \cdot 2^{-n}} \phi(\Gamma(t)) d t
$$

So

$$
\widehat{d \mu_{n}}(\xi)=2^{n} \int_{2^{-n}}^{2 \cdot 2^{-n}} e^{i \xi \cdot T(t)} d t
$$

We wish to say in a precise way that $\widehat{d \mu_{n}}(\xi)$ decays as $\xi$ tends to $\infty$ "uniformly" in $n$. (Note that the condition 10) does not imply that $\widehat{d \mu_{n}}(\xi) \rightarrow 0$ as $n \rightarrow \infty$ because $\Gamma(t)$ can contain straight line segments while the condition 11) at least insures that $\Gamma(t)$ is either part of the $\mathrm{x}$-axis or has no straight line segments.

The uniform estimate we obtain is (if 11 ) holds and $\Gamma(t)$ is not a segment of the $\mathrm{x}$-axis

$$
\left|\widehat{d \mu_{n}}(\xi)\right| \leq c \| B^{*}\left(2^{-n}\right) \xi||^{-\varepsilon}
$$

for some $\varepsilon>0$.

The use of the dilation is that we don't have to make a close examination of the curvature of $\Gamma$ in the interval $\left(2^{-n}, 2 \cdot 2^{-n}\right)$, but instead we can "normalize" 
to the interval $(1,2)$. Let us be more precise.

$$
\begin{aligned}
\widehat{d u_{n}}(\xi)=2^{n} \int_{2^{-n}}^{2 \cdot 2^{-n}} e^{i \xi \cdot \Gamma(t)} d t & =\int_{1}^{2} e^{\mathrm{i} \xi \Gamma\left(2^{-n} t\right)} d t \\
& =\int_{1}^{2} e^{i \xi \cdot\left(B\left(2^{-n}\right) \cdot B^{-1}\left(2^{-n}\right) \Gamma\left(2^{-n} t\right)\right)} d t \\
& =\int_{1}^{2} e^{i B^{\cdot}\left(2^{-n}\right) \xi \cdot B^{-1}\left(2^{-n}\right) \Gamma\left(2^{-n} t\right)} d t
\end{aligned}
$$

Thus to prove 13) it suffices to show

$$
\left\|\int_{1}^{2} e^{i \eta \Gamma_{n}(t)} d t \mid \leq C\right\| \eta \|^{-1 / 2}
$$

where $\eta$ is a vector in $R^{2}$ and $\Gamma_{n}(t)=B^{-1}\left(2^{-n}\right) \Gamma\left(2^{-n} t\right)$. A calculation shows that

$$
\Gamma_{n}(t)=\left(t, \gamma_{n}(t)\right)
$$

where $\gamma_{n}(t)$ has the following properties:

$$
\gamma_{n}(t) \text { is convex }
$$

$$
\gamma_{n}^{t}(1)=1 \quad \gamma_{n}(1)=0
$$

$\left(\right.$ Here $\left.h_{n}(s)=s \gamma_{n}^{\prime}(s)-\gamma_{n}(s)\right)$, and

$$
h_{n}^{s}(s) \geq \varepsilon \frac{h_{n}(s)}{s} .
$$

$$
\gamma_{n}^{\prime \prime}(s) \geq \frac{\varepsilon}{4} \frac{\gamma_{n}^{\prime}(s)}{s}
$$

17) follows immediately from 11) white 16), 17), and the convexity of $\gamma_{n}$ imply 18). One cas then prove 14) by adapting the ideas of Van Der Corput, see [SW].

To see 18) note that

$$
\gamma_{n}(t)=\int_{1}^{t} \gamma_{n}^{\prime}(s) d s \leq(t-1) \gamma_{n}^{\prime}(t)
$$

So

$$
\gamma_{n}^{\prime}(t) \leq h_{n}(t) \leq \frac{t}{\varepsilon} h_{n}^{\prime}(t)=\frac{t^{2}}{\varepsilon} \gamma_{n}^{\prime \prime}(t)
$$


Thus for $1 \leq t \leq 2$

$$
\gamma_{n}^{\prime \prime}(t) \geq \frac{\varepsilon}{4} \gamma_{n}^{\prime}(t)
$$

Finally 14) is obtained from Van Der Corput's Lemmas, Lemma 4.3. of [CCVWW]. If $\left|\eta_{2}\right| \geq\left|\eta_{1}\right|$, we use the "second derivative estimate" together with 18) and 16). If $\left|\eta_{1}\right|>\left|\eta_{2}\right|$ we use the "first derivative estimate" for $t^{\prime} s$ such that $\left|\eta_{2}\right| \gamma_{n}^{\prime}(t)<\frac{\left|n_{1}\right|}{2}$ and the "second derivative cstimate" together with 18) for $t^{\prime} s$ such that

$$
\left|\eta_{2}\right| \gamma_{n}^{\prime}(t)>\frac{\left|\eta_{1}\right|}{2}
$$

We remark that the operators considered above are special cases of more general operators where $x$ is in $R^{n}, x-\Gamma(t)$ is replaced by a k-parameter surface $S(x, t),\left(t \in R^{k}\right)$ with $S(x, 0)=x$, and $1 / t$ is replaced by a Calderon Zygmund kernel on $R^{k}$. In this more general setting positive results have been obtained in [C2] and [CNSW] provided $S(x, t)$ satisfies an appropriate curvature condition. We would hope that a better understanding of the operators $H_{\Gamma}$ and $\mathcal{M}_{\Gamma}$ above without the assumption of the curvature condition would eventually carry over to the more general type of operator without assuming the curvature condition. Let us remark that thic arguments in [CNSW] strongly depend on families of dilations.

\section{References}

[CCVWW] A. Carbery, M. Christ, J. Vance, S. Wainger and D. Watson, Operators associated to flat plane curves, Duke Math. J. (to appear).

[CCCDRVWW] H. Carlsson, M. Christ, A. Cordoba, J. DuoandiKoetXea, J.E. Rubio de Francia, J. Vance, S. Wainger and D. WEINBERG, $L^{p}$ estimates for maximal functions and Hilbert transforms along fiat convex curves in $R^{2}$, Bull. Amer. Math. Soc. 14 (1986), 263-267.

[CW] H. CARLSSON AND S. WAINGER, Maximal functions related to convex polygonal lines, Indiana Univ. Math. J. 34 (1985), 815-823.

[C1] M. CHrist (to appear).

[C2] M. CHinsT, preprint 1985.

[CNSW] M. Chris't, A. NAGEL. E.M. STEin AND S. WAINGER (to appear).

[CW] R. Colfman AND G. WeIss, "Analyse harmonique non-commutative sur certains espaces homogenes," Lecture Notes in Mathematics 242, Springer-Verlag, New York, 1971.

[DR] J. Duoandikontxea and J.L. Rubio de Francia, Maximal and singular integral operators via Fourier transform estimates, Inven. Math. 84 (1986), 541-561. 
[NSW] A. NAgel. E.M. Stein and S. Wainger, Differentiation in lacunary directions, Proc. Natl. Acad. Sci. 75, USA (1978), 1060-1062.

[NVWW1] A. NAGEI, J. VANCE. S. WAinger aND D. WeINBerG, Hilbert transforms for convex curves, Duke Math. J. 50 (1983), 735-744.

[NVWW2] A. NAGEL, J. Vance. S. Wainger and D. Weingerg, Maximal functions for convex curves, Duke Math. J. 52 (1985), 715-722.

[SW] E.M. STEIN AND S. WAINGER, Problems in harmonic analysis related to curvature, Bull. Amer. Math. Soc. 84 (1978), 1239-1295.

Department of Mathematics

University of Wisconsin-Madison

Madison, WI 53706

U.S.A. 